\title{
Effect of magnetic field on the blood flow in artery having multiple stenosis: a numerical study
}

\author{
Gaurav Varshney ${ }^{1}$, V.K. Katiyar ${ }^{2}$, Sushil Kumar ${ }^{3}$ \\ ${ }^{1 *}$ Department of Mathematics, Government Degree College Karanprayag,Chamoli, INDIA \\ ${ }^{2}$ Department of Mathematics,Indian Institute of Technology Roorkee, INDIA \\ Department of Mathematics,S.V. National Institute of Technology Surat, INDIA \\ "Corresponding Author: e-mail: gauravdips@gmail.com, Tel +91-9456717427, Fax.+91-1363-244129
}

\begin{abstract}
In the present study a mathematical model for the blood flow in stenosed artery in the presence of magnetic field is proposed. The laminar, incompressible, fully developed, non-Newtonian flow of blood in an artery having multiple stenosis is numerically studied under the action of transverse magnetic field. The governing equations are transformed by using a radial transformation and the numerical results are obtained using a finite difference technique. Effect of overlapping stenosis and externally applied magnetic field in the blood flow is discussed with the help of graph. All the flow characteristics are found to be affected by the presence of multiple stenosis and exposure of magnetic field of different intensities.
\end{abstract}

Keywords: Blood flow, Magnetic Field, Overlapping Stenosis, generalized Power Law, Finite Difference Method

\section{Introduction}

Many cardiovascular diseases, particularly atherosclerosis, have been found to be responsible for deaths in both developed and developing countries. The study of blood flow through a stenotic artery is very important because the nature of blood movement and mechanical behaviour of vessel walls are causes of many cardiovascular diseases. Most of the authors (Bali and Awasthi, 2007; Chakravarty, 1987; Deplano and Siouffi, 1987; Haldar, 1985; Liu et al., 2004; Mandal, 2005; Shalman et al., 2002) studied the pulsatile blood flow in the artery having single mild stenosis. The multiple stenosis is commonly found in the femoral and pulmonary arteries, so the problem of blood flow becomes more acute in the presence of overlapping stenosis (Chakravarty and Mandal, 1994; Ismail et al., 2007). The study of blood rheology and blood flow has several objectives such as not only understanding health and disease but also in essence, what kind of fluid it is (Buchanan et al., 2000, Hernan and Gonzalez, 2007, Ismail et al., 2007, Mandal, 2005). Some authors in the area of blood flow feel that blood can be assumed to be Newtonian in nature especially in large blood vessels such as the aorta (Katiyar and Vasarajappa, 2002; Liepsch, 2002; Liu et al., 2004; Prakash et al., 2004; Tashtoush and Magableh, 2008; Tzirtzilakis, 2005). In fact blood is a suspension of cells in plasma. The plasma which is a solution of proteins, electrolyte and other substances, is an incompressible virtually Newtonian fluid. From biomechanical point of view, blood is considered as an intelligent fluid, probably the most one in the nature, capable of adapting itself in a great extent in order to provide nutrients to the organs.

It is well known that blood behaves differently when flowing in large vessels, in which Newtonian behaviour is expected and in medium and small vessels where non-Newtonian effects appear (Buchanan et al., 2000; Chakravarty and Mandal, 1994; Deplano and Siouffi, 1987; Ismail et al., 2007; Mandal, 2005). Blood can be regarded as magnetic fluid, in which red blood cells are magnetic in nature. Liquid carriers in the blood contain the magnetic suspension of the particle (Tzirtzilakis, 2005).

Human body experiences magnetic fields of moderate to high intensity in many situations of day to day life. In recent times, many medical diagnostic devices especially those used in diagnosing cardiovascular disease make use of magnetic fields.

It is known from the magneto-hydrodynamics that when a stationary, transverse magnetic field is applied externally to a moving electrically conducting fluid, electrical currents are induced in the fluid. The interaction between these induced currents and the 
applied magnetic field produces a body forces (known as the Lorentz force) which tends to retard the movement of blood (Sud and Sekhon, 1989)

Chester (1957) analyzed the interaction of the magnetic field with the electric current to propose the pressure of body forces in stokes problem for the discussion of the motion of the fluids in the classical hydrodynamics. Low frequency, low intensity magnetic energy has been employed for treating chronic pain secondary to tissue ischemia and slow healing and non healing ulcers with satisfactory to excellent results. This type of energy appears to affect biological process, not through heat production but through electrically induced changes in the environment of cells within the organism.

Jauchem (1997) studied the effects of low frequency electromagnetic energy on the peripheral blood circulation and concluded that low frequency, low intensity magnetic field increased blood flow in the great majority.

Hypertension is one flight mechanics presumably exposed to radio frequency radiation at a level of 38 times above the permissible exposure limit. There are a number of emerging technologies involving the use of Electro-Magnetic frequencies including new types of cellular telephones, magnetically levitated trains and superconducting magnetic energy storage. The possible effects of these particular Electro-Magnetic frequencies on health have not been studied directly.

Magnetic Resonance Imaging is a tool to study the blood flow phenomena in which magnetic field of large intensity is applied on the body. Although existing guidelines on Magnetic Resonance Imaging magnetic fields have been adequate to preclude any known biological problem to date, the Magnetic Resonance Imaging industry would like to have greater flexibility in developing future designs. Simunic et al. used a model to simulate exposure of the human torso to switched magnetic field that would be present during Magnetic Resonance Imaging (Jauchem, 1997)

Villoresi et al. (1994) proposed that when magnetic field exceed $0.05 \mathrm{~T}$, it leads to real dangers connected with development of heart fibrillation and further irreversible changes. He reported a large decrease in myocardial infarction rates on Saturday, Sundays and public holidays and remarked that there were greater man made magnetic disturbances on workdays than during weekends. Myocardial infarctions could be triggered by man made magnetic fields.

Time varying electromagnetic field produced by electrical currents are used to treat non-unified bone fractures. With regards to sympathetic function, time varying electromagnetic fields can modify electrical activity in the brain. This change in electrical activity of the central nervous system can inhibit muscle sympathetic nerve activity and increase skin sympathetic nerve activity (Kinouchi et al., 1996; Kuipers et al., 2007).

Magnetic devices sold to patients commonly utilize static magnetic fields generated by permanent magnets and not time varying electromagnetic fields. Like time varying electromagnetic field, there is some evidence to suggest that static magnetic fields alter autonomic function in human. A $2.0 \mathrm{~T}$ static magnetic field can increase cardiac cycle length, which may be caused by changes within the sinus node. A $0.4 \mathrm{~T}$ static magnetic field can alter skin blood flow in humans, possibly caused by alteration in calcium dynamics (Bali and Awasthi, 2007; Chester, 1957; Gmitrov, 2007; Jauchem, 1997; McKay et al., 2007).

Kuipers et al. (2007) studied the influence of static magnetic fields on cardiovascular and sympathetic function at rest and during physiological stress and also investigated the influence of static magnetic field on pain perception during noxious stimuli.

The biological effects of Magnetic fields have often been linked to nitric oxide (NO), which is responsible for the changes in vessel diameter following magnetic field exposure. Recently magnetic fields have been shown to have positive effects on numerous human systems. For instance, it is documented that magnetic field exposure can provide analgesia, decrease healing time for fractures, increase the speed of nerve regeneration, act as a treatment for depression and provide other medical benefits (McKay et al., 2007).

Magnetic force therapy could be useful for the reperfusion of ischemic tissue or during sepsis. When blood flow to a tissue becomes blocked or reduced, necrosis will eventually occur. Local exposure of a magnetic field could potentially result in blood vessel relaxation and increased blood flow. The effects of magnetism on blood vessels and the cardiovascular system are very interesting. There is still no experiment that shows the effect of quite magnetic field on blood circulation.

In recent years some studies (Katiyar and Basavarajappa, 2002; Kinouchi et al., 1996; Sud and Sekhon, 1989; Tashtoush and Magableh, 2008; Tzirtzilakis, 2005) have been reported on the analysis of blood flow through single arteries in the presence of externally applied magnetic field. However there are very few studies focusing on the effect of magnetic field in the stenotic artery.

Considering the influence of magnetic field on the stenotic artery, in this study, we look at the effect of transverse magnetic field and multi-stenosis on the blood flow in blood vessel. It is assumed that the arterial segment is cylindrical tube with time dependent multi-stenosis and the flowing blood is characterized by generalized Power-law model. Governing equations are solved by using suitable finite difference method. The effect of externally applied magnetic field on velocity, flow rate, flow resistance and wall shear stress is studied

\section{Formulation of the problem}

\subsection{Mathematical model}

It is well known that blood acts as an electrically conducting fluid. When solid material as $F_{e}$ is moving in a magnetic field, it experiences an electromotive force and as a result an electric current may flow. If we apply a magnetic field on an electrically 
conducting fluid like blood, an electromagnetic force will be produced due to the interaction of current with magnetic field. The electromotive force is proportional to the speed of motion and the magnetic flux intensity B (Tashtoush and Magableh, 2008)

Maxwells relations are performed as follows:

The current density $\mathrm{J}$ is expressed by

$\mathrm{J}=\sigma(\mathrm{E}+\mathrm{v} \times \mathrm{B})$

Where $\mathrm{E}$ is the electric field intensity, $\sigma$ is the electrical conductivity, $\mathrm{B}$ is the magnetic flux intensity and $\mathrm{v}$ is the velocity vector. In the momentum equation, the electromagnetic force $\mathrm{F}_{\mathrm{m}}$ is included and is defined as

$\mathrm{F}_{\mathrm{m}}=\mathrm{J} \times \mathrm{B}=\sigma(\mathrm{v} \times \mathrm{B}) \times \mathrm{B}$

The Navier Stokes equations for blood flow including a Lorentz force is

$\rho\left(\frac{\partial \mathrm{v}}{\partial \mathrm{t}}+\mathrm{v} \cdot \nabla \mathrm{v}\right)=\sigma(\mathrm{v} \times \mathrm{B}) \times \mathrm{B}-\nabla \mathrm{p}+\mu \nabla^{2} \mathrm{v}$

Where $\rho$ is the density, $\mu$ is viscosity and $p$ is the pressure.

The artery having stenosis is considered as a cylindrical elastic tube of the circular cross section containing an incompressible non-Newtonion fluid. The blood flow is modeled to be laminar, unsteady, two-dimensional, axi-symmetric and fully developed. Blood is characterized by generalized Power-law model. The flow is considered to take place under the influence of externally applied magnetic field in axial direction. Under these assumptions, the governing equations may be written in the cylindrical coordinates system $(\mathrm{r}, \mathrm{z}, \theta)$ as

Equation of continuity

$\frac{\partial \mathrm{v}_{\mathrm{r}}}{\partial \mathrm{r}}+\frac{\mathrm{v}_{\mathrm{r}}}{\mathrm{r}}+\frac{\partial \mathrm{v}_{\mathrm{z}}}{\partial \mathrm{z}}=0$

Equation of axial momentum

$\frac{\partial \mathrm{v}_{\mathrm{z}}}{\partial \mathrm{t}}+\mathrm{v}_{\mathrm{r}} \frac{\partial \mathrm{v}_{\mathrm{z}}}{\partial \mathrm{r}}+\mathrm{v}_{\mathrm{z}} \frac{\partial \mathrm{v}_{\mathrm{z}}}{\partial \mathrm{z}}=\frac{1}{\rho} \frac{\partial \mathrm{p}}{\partial \mathrm{z}}-\frac{1}{\rho}\left[\frac{1}{\mathrm{r}} \frac{\partial}{\partial \mathrm{r}}\left(\mathrm{r} \sigma_{\mathrm{rz}}\right)+\frac{\partial}{\partial \mathrm{z}}\left(\sigma_{\mathrm{zz}}\right)\right]-\frac{\sigma \mathrm{B}^{2}}{\rho} \mathrm{v}_{\mathrm{z}}$

Equation of radial momentum

$\frac{\partial \mathrm{v}_{\mathrm{r}}}{\partial \mathrm{t}}+\mathrm{v}_{\mathrm{r}} \frac{\partial \mathrm{v}_{\mathrm{r}}}{\partial \mathrm{r}}+\mathrm{v}_{\mathrm{z}} \frac{\partial \mathrm{v}_{\mathrm{r}}}{\partial \mathrm{z}}=-\frac{1}{\rho} \frac{\partial \mathrm{p}}{\partial \mathrm{r}}-\frac{1}{\rho}\left[\frac{1}{\mathrm{r}} \frac{\partial}{\partial \mathrm{r}}\left(\mathrm{r} \sigma_{\mathrm{rr}}\right)+\frac{\partial}{\partial \mathrm{z}}\left(\sigma_{\mathrm{rz}}\right)\right]$

Where the relationship between the shear stress and the shear rate in case of two dimensional motions are given by (Gerrard and Taylor, 1977)

$\sigma=-\mathrm{m}\left[\left|\sqrt{\frac{1}{2}(\dot{\gamma} \cdot \dot{\gamma})}\right|^{\mathrm{n}-1}\right] \gamma$

With $\frac{1}{2}(\dot{\gamma} \cdot \dot{\gamma})=2\left\{\left(\frac{\partial \mathrm{v}_{\mathrm{r}}}{\partial \mathrm{r}}\right)^{2}+\left(\frac{\mathrm{v}_{\mathrm{r}}}{\mathrm{r}}\right)^{2}+\left(\frac{\partial \mathrm{v}_{\mathrm{z}}}{\partial \mathrm{z}}\right)^{2}\right\}+\left(\frac{\partial \mathrm{v}_{\mathrm{r}}}{\partial \mathrm{z}}+\frac{\partial \mathrm{v}_{\mathrm{z}}}{\partial \mathrm{r}}\right)^{2}$

Here $\sigma$ denotes the stress tensor, $\dot{\gamma}$, the symmetric rate of deformation tensor, $\mathrm{m}$ and $\mathrm{n}$ are the respective consistency and fluid behavior parameters

$\sigma_{\mathrm{zz}}=-2\left\{\left.\mathrm{~m}\left[\left(\frac{\partial \mathrm{v}_{\mathrm{r}}}{\partial \mathrm{r}}\right)^{2}+\left(\frac{\mathrm{v}_{\mathrm{r}}}{\mathrm{r}}\right)^{2}+\left(\frac{\partial \mathrm{v}_{\mathrm{z}}}{\partial \mathrm{z}}\right)^{2}+\left(\frac{\partial \mathrm{v}_{\mathrm{r}}}{\partial \mathrm{z}}+\frac{\partial \mathrm{v}_{\mathrm{r}}}{\partial \mathrm{r}}\right)^{2}\right]^{1 / 2}\right|^{\mathrm{n}-1}\right\}\left(\frac{\partial \mathrm{v}_{\mathrm{z}}}{\partial \mathrm{z}}\right)$,
$\sigma_{\mathrm{rz}}=-\left\{\left.\mathrm{m}\left[\left(\frac{\partial \mathrm{v}_{\mathrm{r}}}{\partial \mathrm{r}}\right)^{2}+\left(\frac{\mathrm{v}_{\mathrm{r}}}{\mathrm{r}}\right)^{2}+\left(\frac{\partial \mathrm{v}_{\mathrm{z}}}{\partial \mathrm{z}}\right)^{2}+\left(\frac{\partial \mathrm{v}_{\mathrm{r}}}{\partial \mathrm{z}}+\frac{\partial \mathrm{v}_{\mathrm{z}}}{\partial \mathrm{r}}\right)^{2}\right]^{1 / 2}\right|^{\mathrm{n}-1}\right\}\left(\frac{\partial \mathrm{v}_{\mathrm{z}}}{\partial \mathrm{r}}+\frac{\partial \mathrm{v}_{\mathrm{r}}}{\partial \mathrm{z}}\right)$

and

$\sigma_{\mathrm{rr}}=-2\left\{\mathrm{~m}\left|\left[\left(\frac{\partial \mathrm{v}_{\mathrm{r}}}{\partial \mathrm{r}}\right)^{2}+\left(\frac{\mathrm{v}_{\mathrm{r}}}{\mathrm{r}}\right)^{2}+\left(\frac{\partial \mathrm{v}_{\mathrm{z}}}{\partial \mathrm{z}}\right)^{2}+\left(\frac{\partial \mathrm{v}_{\mathrm{r}}}{\partial \mathrm{z}}+\frac{\partial \mathrm{v}_{\mathrm{z}}}{\partial \mathrm{r}}\right)^{2}\right]^{1 / 2}\right|^{\mathrm{n}-1}\right\}\left(\frac{\partial \mathrm{v}_{\mathrm{r}}}{\partial \mathrm{r}}\right)$

Here $v_{z}(r, z, t)$ and $v_{r}(r, z, t)$ represents the axial and the radial velocity components respectly $p$ is the pressure and $\rho$ is the 
density of blood. The pressure gradient $\partial \mathrm{p} / \partial \mathrm{z}$ appearing in equation (5), is given by

$-\frac{\partial \mathrm{p}}{\partial \mathrm{z}}=\mathrm{A}_{0}+\mathrm{A}_{1} \operatorname{Cos} \omega \mathrm{t}, \mathrm{t}>0$.

Where $\mathrm{A}_{0} \rightarrow$ constant amplitude of the pressure gradient

$A_{1} \rightarrow$ amplitude of the pulsatile component giving rise to systolic and diastolic pressure and $\omega=2 \pi f_{p}$, where $f_{p}$ is the pulse frequency .

\subsection{Boundary conditions}

(1) There is no radial flow along the axis of the artery and the axial velocity gradient of the streaming blood may be assumed to be equal to zero i.e., there is no shear rate of fluid along the axis.

$\mathrm{v}_{\mathrm{r}}(\mathrm{r}, \mathrm{z}, \mathrm{t})=0, \frac{\partial \mathrm{v}_{\mathrm{z}}(\mathrm{r}, \mathrm{z}, \mathrm{t})}{\partial \mathrm{r}}=0$ and $\sigma_{\mathrm{rz}}=0$ at $\mathrm{r}=0$

(2) The velocity on the artery wall is taken as

$\mathrm{v}_{\mathrm{r}}(\mathrm{r}, \mathrm{z}, \mathrm{t})=\frac{\partial \mathrm{R}}{\partial \mathrm{t}}$ and $\mathrm{v}_{\mathrm{z}}(\mathrm{r}, \mathrm{z}, \mathrm{t})=0$ at $\mathrm{r}=\mathrm{R}(\mathrm{z}, \mathrm{t})$

(3) It is assumed that no flow takes places when the system is at rest.

$\mathrm{v}_{\mathrm{r}}(\mathrm{r}, \mathrm{z}, \mathrm{t})=0$ and $\mathrm{v}_{\mathrm{z}}(\mathrm{r}, \mathrm{z}, \mathrm{t})=0$ at $\mathrm{t}=0$

\subsection{Geometry of stenosed artery}

The multiple stenosis is commonly found in the femoral and pulmonary arteries. The geometry of the arterial segment having multiple stenosis is mathematically given by (Chakarvarty and Mandal, 1994)

$R(z, t)=\left\{\begin{array}{cc}a\left(1-a_{1}(t)\left[\frac{11}{32}(z-d) 1^{3}-\frac{47}{48}(z-d)^{2} 1^{2}+(z-d)^{3} 1-\frac{1}{3}(z-d)^{4}\right]\right), & d \leq z \leq d+\frac{31}{2} \\ \text { a. } a_{1}(t), & ; \text { otherwise }\end{array}\right\}$

where $a$ is the unconstricted radius of stenosed artery, $d$ is the location of stenosis and 1 is the length of the stenosis.

The time variant parameter is given by $a_{1}(t)=1-b \operatorname{Cos}(\omega t-1) e^{-b \omega t} ; \omega=2 \pi f_{p}$.

\section{Solution procedure}

\subsection{Transformation of the governing equations}

Let us introduce a radial coordinate transformation $\left[\mathrm{x}=\frac{\mathrm{r}}{\mathrm{R}(\mathrm{z}, \mathrm{t})}\right]$, which has the effect of immobilizing the vessel wall in the transformed coordinate $\mathrm{x}$.

Using this transformation, equations (4-8) together with boundary conditions (10-12) take the following form : -

$\frac{1}{\mathrm{r}} \frac{\partial \mathrm{v}_{\mathrm{r}}}{\partial \mathrm{x}}+\frac{\mathrm{v}_{\mathrm{r}}}{\mathrm{xR}}+\frac{\partial \mathrm{v}_{\mathrm{z}}}{\partial \mathrm{z}}-\frac{\mathrm{x}}{\mathrm{R}} \frac{\partial \mathrm{v}_{\mathrm{z}}}{\partial \mathrm{x}} \frac{\partial \mathrm{R}}{\partial \mathrm{z}}=0$

$\frac{\partial \mathrm{v}_{\mathrm{z}}}{\partial \mathrm{t}}=\left[\frac{\mathrm{x}}{\mathrm{R}} \frac{\partial \mathrm{R}}{\partial \mathrm{t}}-\frac{\mathrm{v}_{\mathrm{r}}}{\mathrm{R}}+\mathrm{v}_{\mathrm{z}} \frac{\mathrm{x}}{\mathrm{R}} \frac{\partial \mathrm{R}}{\partial \mathrm{z}}\right] \frac{\partial \mathrm{v}_{\mathrm{z}}}{\partial \mathrm{x}}-\mathrm{v}_{\mathrm{z}} \frac{\partial \mathrm{v}_{\mathrm{z}}}{\partial \mathrm{z}}-\frac{1}{\rho} \frac{\partial \mathrm{p}}{\partial \mathrm{z}}-$

$\frac{1}{\rho}\left[\frac{1}{x R} \sigma_{x z}+\frac{1}{R} \frac{\partial \sigma_{x z}}{\partial x}+\frac{\partial \sigma_{z z}}{\partial z}-\frac{x}{R} \frac{\partial \sigma_{x z}}{\partial x} \frac{\partial R}{\partial z}\right]-M_{z}$

Where $M=\frac{\sigma B^{2}}{\rho}$

Normal stress 


$$
\begin{aligned}
\sigma_{\mathrm{zz}}=-2\left\{\mathrm{~m}\left|\left[\left(\frac{1}{\mathrm{R}} \frac{\partial \mathrm{v}_{\mathrm{r}}}{\partial \mathrm{x}}\right)^{2}+\left(\frac{\mathrm{v}_{\mathrm{r}}}{\mathrm{xR}}\right)^{2}+\left(\frac{\partial \mathrm{v}_{\mathrm{z}}}{\partial \mathrm{z}}-\frac{\mathrm{x}}{\mathrm{R}} \frac{\partial \mathrm{R}}{\partial \mathrm{z}} \frac{\partial \mathrm{v}_{\mathrm{z}}}{\partial \mathrm{x}}\right)^{2}+\left(\frac{\partial \mathrm{v}_{\mathrm{r}}}{\partial \mathrm{z}}-\frac{\mathrm{x}}{\mathrm{R}} \frac{\partial \mathrm{R}}{\partial \mathrm{z}} \frac{\partial \mathrm{v}_{\mathrm{r}}}{\partial}+\frac{1}{\mathrm{r}} \frac{\partial \mathrm{v}_{2}}{\partial \mathrm{x}}\right)^{2}\right]^{1 / 2}\right|^{\mathrm{n}-1}\right\} \\
\left(\frac{\partial \mathrm{x}_{\mathrm{z}}}{\partial \mathrm{z}}-\frac{\mathrm{x}}{\mathrm{R}} \frac{\partial \mathrm{R}}{\partial \mathrm{z}} \frac{\partial \mathrm{v}_{\mathrm{z}}}{\partial \mathrm{x}}\right)
\end{aligned}
$$

Shear stress

$$
\begin{aligned}
\sigma_{\mathrm{xz}}= & -\left\{\mathrm{m}\left|\left[\left(\frac{1}{\mathrm{R}} \frac{\partial \mathrm{v}_{\mathrm{r}}}{\partial \mathrm{x}}\right)^{2}+\left(\frac{\mathrm{v}_{\mathrm{r}}}{\mathrm{xR}}\right)^{2}+\left(\frac{\partial \mathrm{v}_{\mathrm{z}}}{\partial \mathrm{z}}-\frac{\mathrm{x}}{\mathrm{R}} \frac{\partial \mathrm{R}}{\partial \mathrm{z}} \frac{\partial \mathrm{v}_{\mathrm{z}}}{\partial \mathrm{x}}\right)^{2}+\left(\frac{\partial \mathrm{v}_{\mathrm{r}}}{\partial \mathrm{z}}-\frac{\mathrm{x}}{\mathrm{R}} \frac{\partial \mathrm{R}}{\partial \mathrm{z}} \frac{\partial \mathrm{v}_{\mathrm{r}}}{\partial \mathrm{x}}+\frac{1}{\mathrm{r}} \frac{\partial \mathrm{v}_{\mathrm{z}}}{\partial \mathrm{x}}\right)^{2}\right]^{1 / 2}\right|^{\mathrm{n}-1}\right\} \\
& \left(\frac{1}{\mathrm{R}} \frac{\partial \mathrm{v}_{\mathrm{z}}}{\partial \mathrm{z}}+\frac{\partial \mathrm{v}_{\mathrm{r}}}{\partial \mathrm{z}}-\frac{\mathrm{x}}{\mathrm{R}} \frac{\partial \mathrm{R}}{\partial \mathrm{z}} \frac{\partial \mathrm{v}_{\mathrm{z}}}{\partial \mathrm{x}}\right),
\end{aligned}
$$

where $\mathrm{n}$ is the fluid behavior index parameter

3.2 Transformation of the Boundary Conditions

$\mathrm{v}_{\mathrm{r}}(\mathrm{x}, \mathrm{z}, \mathrm{t})=0, \frac{\partial \mathrm{v}_{\mathrm{z}}(\mathrm{x}, \mathrm{z}, \mathrm{t})}{\partial \mathrm{r}}=0$ and $\sigma_{\mathrm{xz}}=0$ an $\mathrm{x}=0$

$\mathrm{v}_{\mathrm{r}}(\mathrm{x}, \mathrm{z}, \mathrm{t})=\frac{\partial \mathrm{R}}{\partial \mathrm{t}}$ and $\mathrm{v}_{\mathrm{z}}(\mathrm{x}, \mathrm{z}, \mathrm{t})=0$ on $\mathrm{x}=1$ and

$\mathrm{v}_{\mathrm{r}}(\mathrm{x}, \mathrm{z}, \mathrm{t})=0$ and $\mathrm{v}_{\mathrm{z}}(\mathrm{x}, \mathrm{z}, \mathrm{t})=0$ at $\mathrm{t}=0$.

In order to get the radial velocity component, $\mathrm{v}_{\mathrm{r}}(\mathrm{x}, \mathrm{z}, \mathrm{t})$, we have to consider the equation (4) as

$\mathrm{x} \frac{\partial \mathrm{v}_{\mathrm{r}}}{\partial \mathrm{x}}+\mathrm{v}_{\mathrm{r}}+\mathrm{xR} \frac{\partial \mathrm{v}_{\mathrm{z}}}{\partial \mathrm{z}}-\mathrm{x}^{2} \frac{\partial \mathrm{v}_{\mathrm{z}}}{\partial \mathrm{x}} \cdot \frac{\partial \mathrm{R}}{\partial \mathrm{z}}=0$

Now, integrating the equation (21) with respect to $\mathrm{x}$ from the limits $0 \rightarrow \mathrm{x}$, we get (Mandal, 2005):

$\mathrm{v}_{\mathrm{r}}(\mathrm{x}, \mathrm{z}, \mathrm{t})=\mathrm{x}\left[\frac{\partial \mathrm{R}}{\partial \mathrm{z}} \mathrm{v}_{\mathrm{z}}-\frac{\partial \mathrm{R}}{\partial \mathrm{t}}\left(\mathrm{z}-\mathrm{x}^{2}\right)\right]$

\subsection{Discretization of the axial velocity component, $\mathrm{v}_{\mathrm{z}}(\mathrm{x}, \mathrm{z}, \mathrm{t})$}

The discretization of axial velocity $v_{z}(x, z, t)$ is written as $v_{z}\left(x_{j}, z_{i}, t_{k}\right)$ or $\left(v_{z}\right)_{i, j}^{k}$

We define

$\mathrm{x}_{\mathrm{j}}=\mathrm{j} . \Delta \mathrm{x} ; \mathrm{j}=0,1,2 \ldots \mathrm{N}$ where $\mathrm{x}_{\mathrm{N}}=1.0$

$\mathrm{z}_{\mathrm{i}}=\mathrm{i} . \Delta \mathrm{z} ; \mathrm{i}=0,1,2 \ldots \mathrm{M}$

$\mathrm{t}_{\mathrm{K}}=(\mathrm{K}-1) \Delta \mathrm{t} ; \mathrm{K}=1,2, \ldots$

The Finite difference scheme is used to solve the governing transformed equation by using central difference approximations for all the spatial derivatives in the following manner:

$\frac{\partial v_{z}}{\partial x}=\frac{\left(v_{z}\right)_{i, j+1}^{K}-\left(v_{z}\right)_{i, j-1}^{K}}{2 \Delta x}=\left(V_{z}\right)_{f_{x}}$ and $\frac{\partial v_{Z}}{\partial x}=\frac{\left(v_{z}\right)_{i+1, j}^{K}-\left(v_{z}\right)_{i-j, j}^{K}}{2 \Delta z}=\left(v_{z}\right)_{f_{z}}$

and the time derivatives are approximated by

$\frac{\partial \mathrm{v}_{\mathrm{z}}}{\partial \mathrm{t}}=\frac{\left(\mathrm{v}_{\mathrm{z}}\right)_{\mathrm{i}, \mathrm{j}}^{\mathrm{K}+1}-\left(\mathrm{v}_{\mathrm{z}}\right)_{\mathrm{i}, \mathrm{j}}^{\mathrm{K}}}{2 \Delta \mathrm{t}}$ 
Similarly, the derivatives for $\mathrm{v}_{\mathrm{r}}, \sigma_{\mathrm{zz}}$ and $\sigma_{\mathrm{xz}}$ are

$$
\begin{aligned}
& \frac{\partial v_{r}}{\partial x}=\frac{\left(v_{r}\right)_{i, j+1}^{K}-\left(v_{r}\right)_{i, j-1}^{K}}{2 \Delta x}=\left(v_{r}\right)_{f_{x}} \\
& \frac{\partial v_{r}}{\partial z}=\frac{\left(v_{r}\right)_{i+1, j}^{K}-\left(v_{r}\right)_{i-1, j}^{K}}{2 \Delta z}=\left(v_{r}\right)_{f_{z}} \\
& \frac{\partial \sigma_{z z}}{\partial x}=\frac{\left(\sigma_{z z}\right)_{i, j+1}^{K}-\left(\sigma_{z z}\right)_{i, j-1}^{K}}{2 \Delta x}=\left(\sigma_{z z}\right)_{f_{x}}, \\
& \frac{\partial \sigma_{z z}}{\partial z}=\frac{\left(\sigma_{z z}\right)_{i+1, j}^{K}-\left(\sigma_{z z}\right)_{i-1, j}^{K}}{2 \Delta z}=\left(\sigma_{z z}\right)_{f_{z}}, \\
& \text { and } \frac{\partial \sigma_{x z}}{\partial x}=\frac{\left(\sigma_{x z}\right)_{i, j+1}^{K}-\left(\sigma_{x z}\right)_{i, j-1}^{K}}{2 \Delta x}=\left(\sigma_{x z}\right)_{f_{x}}
\end{aligned}
$$

Using above discritization techniques equation (22-26) for axial velocity, equation (15) may be transformed to following difference equations:

$$
\begin{aligned}
& \left(v_{z}\right)_{i, j}^{K+1}=\left(V_{z}\right)_{i, j}^{K}+\Delta t\left\{\left[\frac{x_{j}}{R_{t}^{k}} \cdot\left(\frac{\partial R}{\partial t}\right)_{i}^{K}-\frac{\left(v_{r}\right)_{i, j}^{K}}{R_{t}^{k}}+\left(v_{z}\right)_{i, j}^{K} \frac{x_{j}}{R_{i}^{K}} \cdot\left(\frac{\partial R}{\partial z}\right)_{i}^{K}\right]\right. \\
& \left(\left(v_{z}\right)_{f x}\right)_{i, j}^{K}-\left(v_{z}\right)_{i, j}^{K}\left(\left(v_{z}\right)_{t z}\right)_{i, j}^{K}-\frac{1}{\rho}\left(\frac{\partial p}{\partial z}\right)^{K+1} \frac{1}{\rho}\left[\frac{1}{x_{i} R_{i}^{K}}\left(\sigma_{x z}\right)_{i, j}^{K}+\frac{1}{R_{i}^{K}}\left[\left(\sigma_{x z} f_{x}\right)\right]_{i, j}^{K}+\left[\left(\sigma_{z z} f_{z}\right)\right]_{i, j}^{K}-\right. \\
& \left.\frac{x_{i}}{R_{j}^{K}}\left[\left(\sigma_{x z} f_{x}\right)\right]_{i, j}^{K}\left(\frac{\partial R}{\partial Z}\right)_{i}^{K}\right]-M\left(v_{z}\right)_{i, j}^{K}
\end{aligned}
$$

The equation (16) and (17) has their discritized form as:

$$
\begin{aligned}
& \left(\sigma_{z z}\right)_{i, j}^{K}=2\left\{m \mid\left[\left(\frac{1}{R_{i}^{K}}\left(\left(v_{r}\right)_{f x}\right)_{i, j}^{K}\right)^{2}+\left(\frac{\left(v_{r}\right)_{i, j}^{K}}{x_{j} R_{i}^{K}}\right)+\right.\right. \\
& \left(\left(\left(v_{z}\right)_{f_{z}}\right)_{i, j}^{K}-\frac{x_{j}}{R_{i}^{K}}\left(\frac{\partial R}{\partial z}\right)_{i}^{K}\left(\left(v_{z}\right)_{f_{x}}\right)_{i, j}^{K}\right)^{2}+ \\
& \left.\left.\left(\left(\left(v_{r}\right)_{f_{z}}\right)_{i, j}^{K}-\frac{x_{j}}{R_{i}^{K}}\left(\frac{\partial R}{\partial z}\right)_{i}^{K}\left(\left(v_{z}\right)_{x}\right)_{i, j}^{K}+\frac{1}{R_{j}^{k}}\left(\left(V_{z}\right)_{f_{x}}\right)_{i, j}^{K}\right)^{2}\right]^{1 / 2}\right\} \\
& \left(\left(\left(v_{z}\right)_{f_{z}}\right)_{i, j}^{K}-\frac{x_{j}}{R_{i}^{K}}\left(\frac{\partial R}{\partial Z}\right)_{i}^{k}\left(\left(v_{z}\right)_{f_{z}}\right)_{i, j}^{K}\right)
\end{aligned}
$$

and

$$
\begin{aligned}
& \left(\sigma_{x z}\right)_{i, j}^{K}=-2\left\{m\left[\left(\frac{1}{R_{i}^{k}}\left(\left(v_{r}\right)_{f_{x}}\right)_{i, j}^{K}\right)^{2}+\left(\frac{\left(v_{r}\right)_{i, j}^{K}}{x_{j} R_{i}^{k}}\right)^{2}\right]+\right. \\
& \left(\left(\left(v_{z}\right)_{f_{z}}\right)_{i, j}^{K}-\frac{x_{j}}{R_{i}^{K}}\left(\frac{\partial R}{\partial z}\right)_{i}^{K}\left(\left(v_{z}\right)_{f_{x}}\right)_{i, j}^{K}\right)^{2}+
\end{aligned}
$$




$$
\begin{aligned}
& \left.\left.\left.\left(\left(\left(v_{x}\right)_{f_{z}}\right)_{i, j}^{K}-\frac{x_{j}}{R_{i}^{K}}\left(\frac{\partial R}{\partial z}\right)_{i}^{K}\right)\left(\left(v_{r}\right)_{f_{x}}\right)_{i_{j} j}^{K}+\frac{1}{R_{i}^{K}}\left(\left(v_{z}\right)_{f_{x}}\right)_{i, j}^{K}\right)^{2}\right]\left.^{1 / 2}\right|^{n-1}\right\} \\
& \left(\left(\left(v_{r}\right)_{f_{z}}\right)_{i, j}^{K}-\frac{x_{j}}{R_{j}^{K}}\left(\frac{\partial R}{\partial z}\right)_{i}^{K}\left(\left(v_{x}\right)_{f_{x}}\right)_{i, j}^{K}+\frac{1}{R_{i}^{K}}\left(\left(v_{z}\right)_{f_{x}}\right)_{i, j}^{K}\right)
\end{aligned}
$$

The boundary conditions in discretized form are as follows:

$$
\left(\mathrm{v}_{\mathrm{r}}\right)_{\mathrm{i}, \mathrm{j}}^{\mathrm{K}}=0,\left(\mathrm{v}_{\mathrm{z}}\right)_{\mathrm{i}, 1}^{\mathrm{K}}=\left(\mathrm{v}_{\mathrm{z}}\right)_{\mathrm{i}, 2}^{\mathrm{K}},\left(\sigma_{\mathrm{xz}}\right)_{\mathrm{i}, 1}^{\mathrm{K}}=0,\left(\mathrm{v}_{\mathrm{z}}\right)_{\mathrm{i}, \mathrm{N}+1}^{\mathrm{K}}=0,\left(\mathrm{v}_{\mathrm{r}}\right)_{\mathrm{i}, \mathrm{N}+1}^{\mathrm{K}}=\left(\frac{\partial \mathrm{R}}{\partial \mathrm{t}}\right)_{\mathrm{i}}^{\mathrm{K}}
$$

and

$$
\left(\mathrm{v}_{\mathrm{r}}\right)_{\mathrm{i}, \mathrm{j}}^{1}=0,\left(\mathrm{v}_{\mathrm{z}}\right)_{\mathrm{i}, \mathrm{j}}^{1}=0
$$

The radial velocity can be calculated from the equation (22) and its discretized form is

$$
\left(\mathrm{v}_{\mathrm{r}}\right)_{\mathrm{i}, \mathrm{j}}^{\mathrm{K}+1}=\mathrm{x}_{\mathrm{j}}\left[\left(\frac{\partial \mathrm{R}}{\partial \mathrm{z}}\right)_{\mathrm{i}}^{\mathrm{K}}\left(\mathrm{v}_{\mathrm{z}}\right)_{\mathrm{i}, \mathrm{j}}^{\mathrm{K}+1}-\left(\frac{\partial \mathrm{R}}{\partial \mathrm{t}}\right)_{\mathrm{i}}^{\mathrm{K}}\left[2-\mathrm{x}_{\mathrm{j}}^{2}\right]\right]
$$

The flow rate can be obtained by

$$
\mathrm{Q}=\int_{0}^{\mathrm{R}} 2 \pi \mathrm{rv}_{\mathrm{z}} \mathrm{dr}
$$

by using the radial coordinate transformation, the discretized form of flow rate $\mathrm{Q}$ is given by

$$
\mathrm{Q}_{\mathrm{i}}^{\mathrm{K}}=2 \pi\left(\mathrm{R}_{\mathrm{i}}^{\mathrm{K}}\right)^{2} \int_{0}^{1} \mathrm{x}\left(\mathrm{v}_{\mathrm{z}}\right)_{\mathrm{i}, \mathrm{j}}^{\mathrm{K}} \mathrm{dx} \mathrm{x}_{\mathrm{j}}
$$

The Resistance of flow $\lambda$ can be calculated from

$$
\lambda=\frac{|\mathrm{L}(\partial \mathrm{p} / \partial \mathrm{z})|}{\mathrm{Q}}
$$

Its discretized form is given by

$$
\lambda_{\mathrm{i}}^{\mathrm{K}}=\frac{\left|\mathrm{L}(\partial \mathrm{p} / \partial \mathrm{z})^{\mathrm{K}}\right|}{\mathrm{Q}_{\mathrm{i}}^{\mathrm{K}}}
$$

The wall share stress is defined as

$$
\tau_{\mathrm{w}}=\mu\left(\frac{\partial \mathrm{v}_{\mathrm{z}}}{\partial \mathrm{x}}+\frac{\partial \mathrm{v}_{\mathrm{x}}}{\partial \mathrm{z}}\right)
$$

In the discretized from equation (41) can be written as

$$
\left(\tau_{\mathrm{w}}\right)_{\mathrm{i}}^{\mathrm{K}}=\mu\left[\frac{1}{\mathrm{R}_{\mathrm{i}}^{\mathrm{K}}}\left(\left(\mathrm{v}_{\mathrm{z}}\right)_{\mathrm{f}_{\mathrm{z}}}\right)_{\mathrm{i}, \mathrm{j}}^{\mathrm{K}}+\left(\left(\mathrm{v}_{\mathrm{r}}\right) \mathrm{f}_{\mathrm{z}}\right)_{\mathrm{i}, \mathrm{j}}^{\mathrm{K}}-\frac{\mathrm{x}_{\mathrm{j}}}{\mathrm{R}_{\mathrm{i}}^{\mathrm{K}}}\left(\left(\mathrm{v}_{\mathrm{r}}\right)_{\mathrm{f}_{\mathrm{x}}}\right)_{\mathrm{i}, \mathrm{j}}^{\mathrm{K}}\left(\frac{\partial \mathrm{R}}{\partial \mathrm{Z}}\right)_{\mathrm{i}}^{\mathrm{K}}\right]_{\mathrm{x}=1} \times \cos \left[\arctan \left(\frac{\partial \mathrm{R}}{\partial \mathrm{z}}\right)_{\mathrm{i}}^{\mathrm{K}}\right]
$$

\section{Result and discussion}

Numerical computations have been carried out using the following parameter values (Chakravarty, 1987, Ismail et al., 2007; Katiyar and Vasarajappa, 2002; Mandal, 2005).

$$
\begin{aligned}
& \mathrm{a}=0.08 \mathrm{~cm}, \mathrm{~L}=5 \mathrm{~cm}, \mathrm{l}_{0}=1.6 \mathrm{~cm}, \mathrm{~d}=1.3 \mathrm{~cm}, \mathrm{~b}=0.1, \mathrm{~m}=0.1735 \mathrm{P}, \mu=0.035 \mathrm{P}, \mathrm{n}=0.639, \\
& \rho=1.06 \mathrm{~g} \mathrm{~cm}^{3}, \mathrm{f}_{\mathrm{p}}=1.2 \mathrm{~Hz}, \mathrm{~A}_{0}=10 \mathrm{~g} \mathrm{~cm}^{-2} \mathrm{~s}^{-2}, \mathrm{~A}_{1}=0.2 \mathrm{~A}_{0}, \tau_{\mathrm{m}}=0.4 * \mathrm{a}, \omega=2.4 * \mathrm{pi}, \\
& \Delta \mathrm{x}=0.02, \Delta \mathrm{z}=0.1 \text { and } \Delta \mathrm{t}=0.00001
\end{aligned}
$$

The result appeared to converge with an accuracy of the order $\sim 10^{-5}$ when the time step was chosen to be $\Delta t=0.00001$.

Figure 1 shows the geometry of the moving arterial wall of the time variant multiple stenosis for different taper angles. It is clear that the radius of artery increases during systole and as the diastolic phase start, the radius of the artery decreases. 


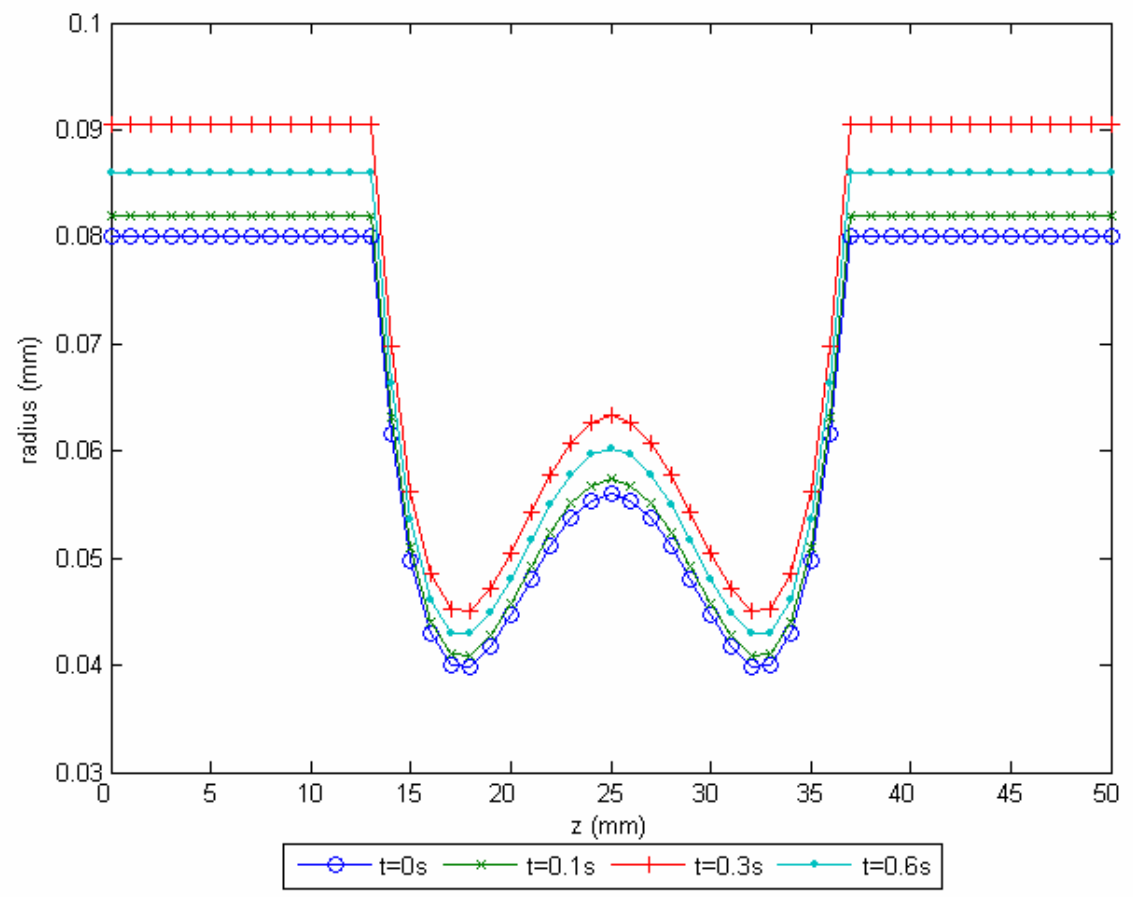

Figure 1. Radius of constricted Artery at different time

The complete axial and radial velocity profile in the artery geometry is shown in Figures 2 and 3, respectively. In order to analyze the flow field insensibly along the arterial segment under study, Figure 4 exhibits the axial velocity profiles at various axial locations for $\mathrm{M}=2$ and $\mathrm{t}=0.3 \mathrm{sec}$. The axial velocity profile is parabolic at the upstream $(\mathrm{z}=10 \mathrm{~mm})$, the area without stenosis, while a flattering trend is followed at the converging section $(\mathrm{z}=15 \mathrm{~mm})$ and subsequently it becomes much blunter at the locations $(\mathrm{z}=24 \mathrm{~mm}$ and $\mathrm{z}=28 \mathrm{~mm})$, the critical height of the stenosis than at the entry. The velocity appears to be enhanced at the diverging section $(\mathrm{z}=35 \mathrm{~mm})$ and finally at the end of the stenosis, the axial velocity profile gets back again into parabolic patterns.

The Figure 5 shows the radial velocity profile at various locations for $t=0.3 \mathrm{sec}$. The radial velocity component varying radially for different axial position, which is found to be increasing from zero on the axis with positive values as one moves away from it and finally to attain some finite value on the wall surface. Radial velocity is higher in magnitude in the area without stenosis and the presence of stenosis slows down radial velocity to a considerable extent.

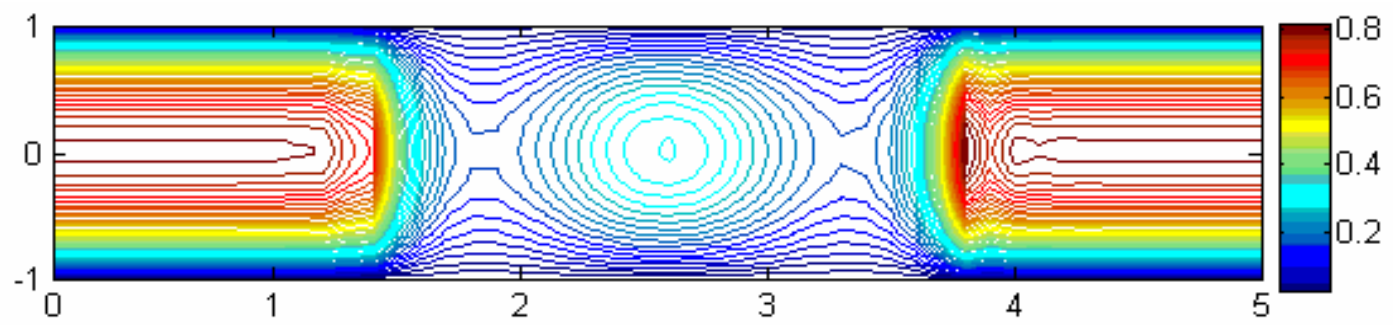

Figure 2 . Axial velocity profile at $\mathrm{t}=0.3 \mathrm{sec}$ in stenosed artery

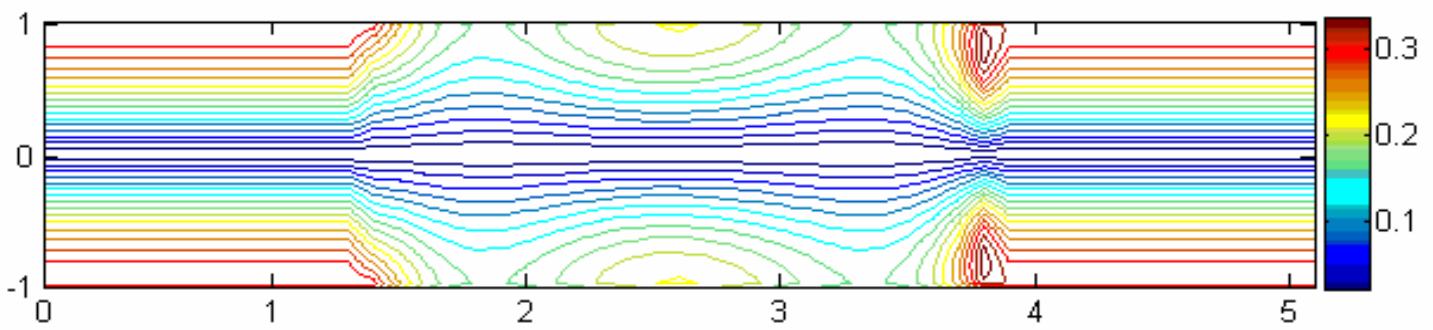

Figure 3 . Radial velocity profile at $\mathrm{t}=0.3 \mathrm{sec}$ in stenosed artery 


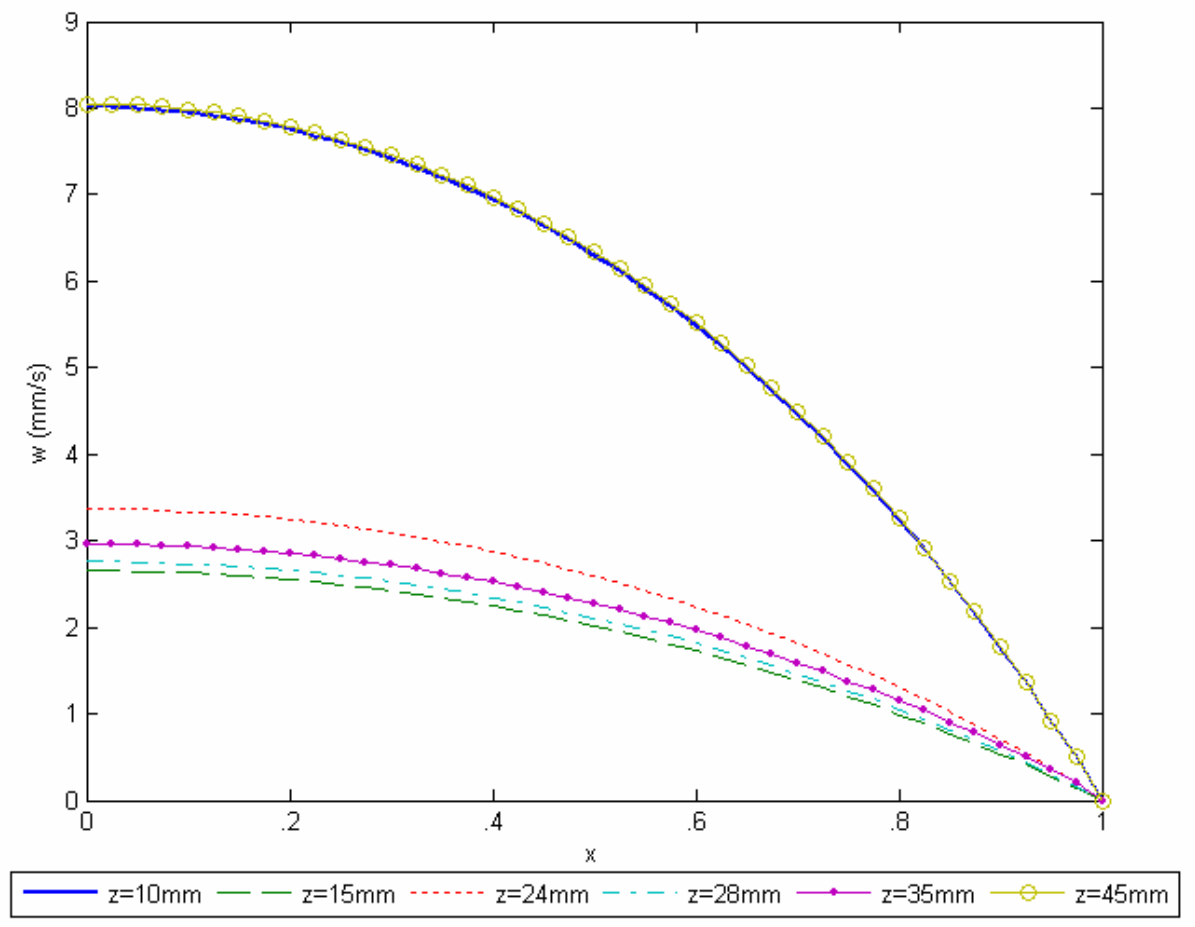

Figure 4. Axial velocity profiles for different axial positions at $\mathrm{t}=0.3 \mathrm{~s}$ $\left(\mathrm{T}_{\mathrm{m}}=0.4 \mathrm{a}, \mathrm{M}=2, \mathrm{~d}=13 \mathrm{~mm}, \mathrm{l}_{0}=16 \mathrm{~mm}\right)$

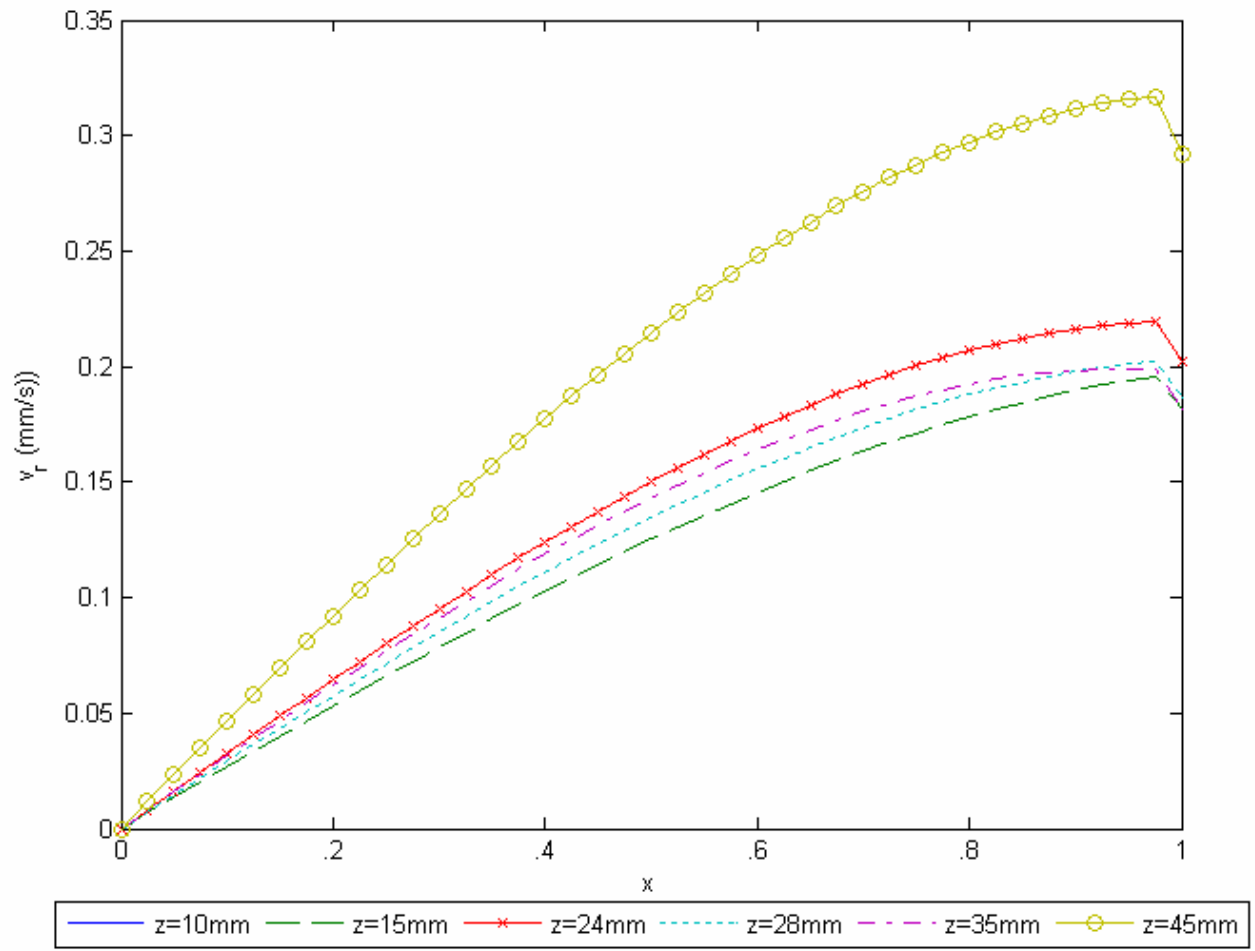

Figure 5. Radial velocity profiles for different axial positions at $\mathrm{t}=0.3 \mathrm{~s}$ $\left(\mathrm{T}_{\mathrm{m}}=0.4 \mathrm{a}, \mathrm{d}=13 \mathrm{~mm}, \mathrm{M}=2, \mathrm{1}_{0}=16 \mathrm{~mm}\right)$ 
Figure 6 shows the variation of the axial velocity profile at $\mathrm{z}=23 \mathrm{~mm}$ for different time periods. The flow profiles are directly responsible for the pulsatile pressure gradient produced by the pumping action of heart. Whenever the time shifted increases from $0.1 \mathrm{sec}$ to $0.3 \mathrm{sec}$, the curve shifts towards the origin. At $\mathrm{t}=0.6 \mathrm{sec}$ again the axial velocity increases because of the diastole. It is also clear that the streaming blood is much higher than the non-Newtonian values. It is because of the fact that if flowing blood is treated as Newtonian, it has a high shear rate flow, which increases the axial velocity profile. Thus the non-Newtonian characteristics of the flowing blood affect the axial velocity profile. The results agrees qualitatively with "Chaturani and Palanisamy (1991) and Sud and Sekhon (1985)" with their research on stenotic blood flow treated as Newtonian fluid.

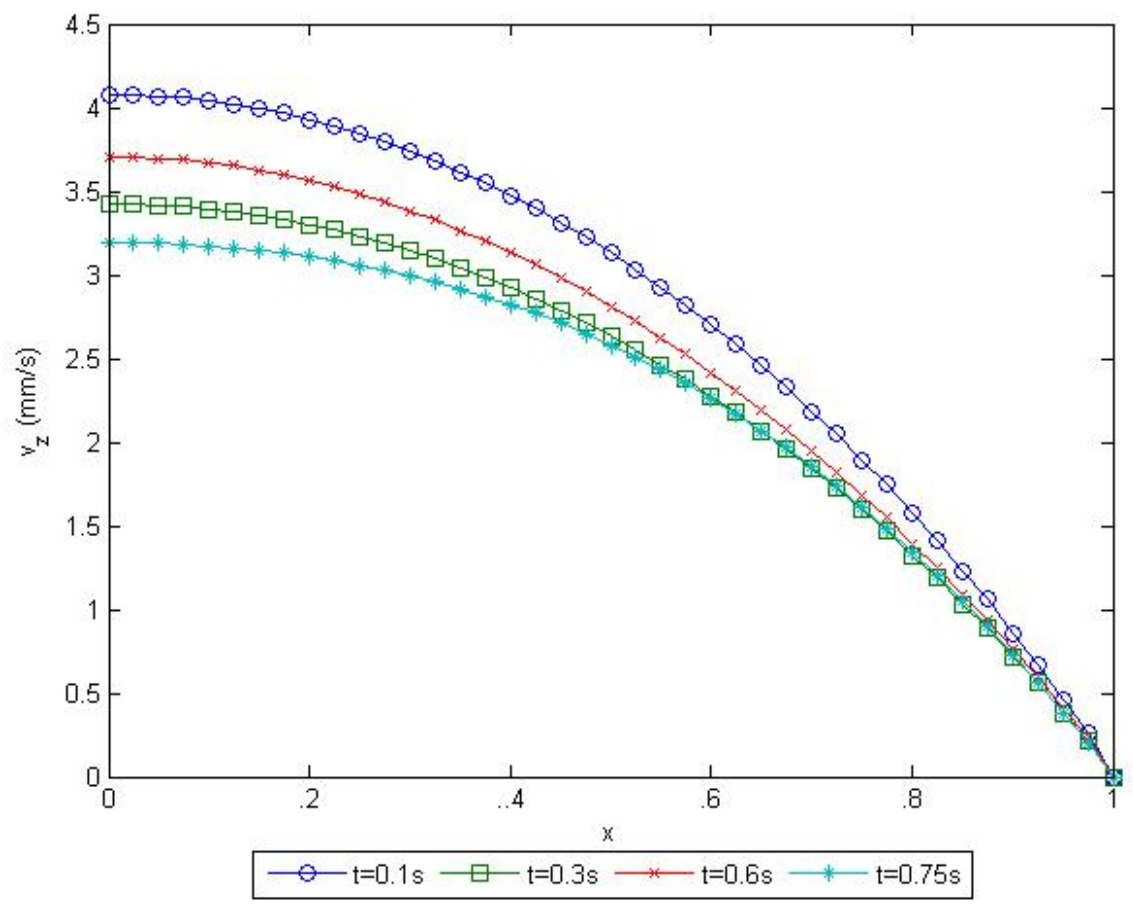

Figure 6. Axial velocity profiles for different times at $\mathrm{z}=23 \mathrm{~mm}$ $\left(\mathrm{T}_{\mathrm{m}}=0.4 \mathrm{a}, \mathrm{d}=13 \mathrm{~mm}, \mathrm{M}=2,1_{0}=16 \mathrm{~mm}\right)$

In Figure 7, the radial velocity profile at different time period is shown. The radial velocity profile assume a positive value during systole i.e. at $\mathrm{t}=0.1 \mathrm{sec}$ and $0.3 \mathrm{sec}$. and negative during diastole i.e. at $\mathrm{t}=0.6 \mathrm{sec}$ and $0.75 \mathrm{sec}$. This is due to the fact that in systolic phase the heart muscle has fully contracted and blood is squeezed out and during diastole, the heart relaxes and becomes full of blood.

Effect of magnetic field intensity on the axial velocity at the specific region $\mathrm{z}=23 \mathrm{~mm}$ and time $\mathrm{t}=0.3 \mathrm{sec}$ is shown in Figure 8 . The higher values of magnetic field intensity reduce the axial velocity in a larger extent. This is due to the fact that as magnetic field applied on the body, the Laurentz force opposes the flow of blood and hence reduces its velocity.

The variation of fluid acceleration $F$ at time $t=0.6 \mathrm{sec}$ at the location $\mathrm{z}=23 \mathrm{~mm}$, where the artery get maximum narrowing is shown in Figure 9. The acceleration is maximum on the axis and attains some finite value on the wall. By increasing the magnetic field intensity, the fluid acceleration decreases rapidly. Blood is accelerated more for the cases without magnetic field and the magnetic field exposure slows down the fluid acceleration.

The variation of flow rate at the location $z=23 \mathrm{~mm}$ over a period of nearly four cardiac cycle for various magnetic field intensity is illustrated in Figure 10. The pulsatile nature of the flow rate has been found in all cases thought the time scale. One may note the flow rate for an artery without applied magnetic field having higher magnitude than the flow rate for artery with applied magnetic field. It is concluded that the presence of magnetic field affect the flow rate to considerable extent.

Figure 11 illustrates the variations of the flow rate $\mathrm{Q}$ with time $\mathrm{t}$ for different values of the frequency and amplitude of pressure gradient for the magnetic field intensity $M=2$ at $z=23 \mathrm{~mm}$. It is clear that by increasing the pulse frequency $f_{p}$, the back flow increases. The magnitude of flow rate increases with increasing $A_{0}$ with positive values i.e. no back flow occurs. Thus the frequency as well as the amplitude of the pressure gradient affects the rate of flow to a considerable extent in the presence of magnetic field. 


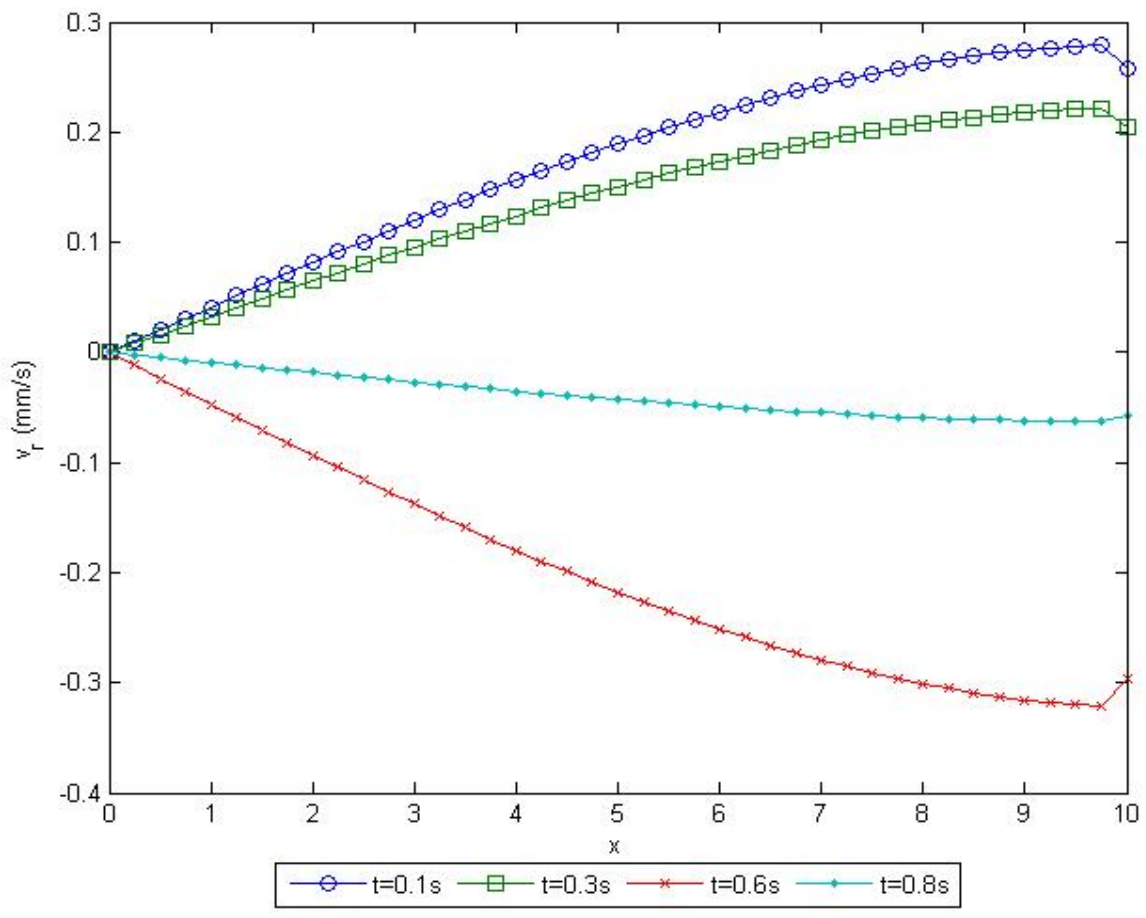

Figure 7. Radial velocity profiles for different times at $\mathrm{z}=23 \mathrm{~mm}$ $\left(\mathrm{T}_{\mathrm{m}}=0.4 \mathrm{a}, \mathrm{d}=13 \mathrm{~mm}, \mathrm{M}=2,1_{0}=16 \mathrm{~mm}\right)$

The variations of the flow resistance with time for different magnetic field are shown in Figure 12. The resistance of flow gives the reverse trend of the flow rate. This is because as the flow rate increases, resistance decreases. The values of resistance are higher for the artery without having applied magnetic field than the artery having the effects of applied magnetic field.

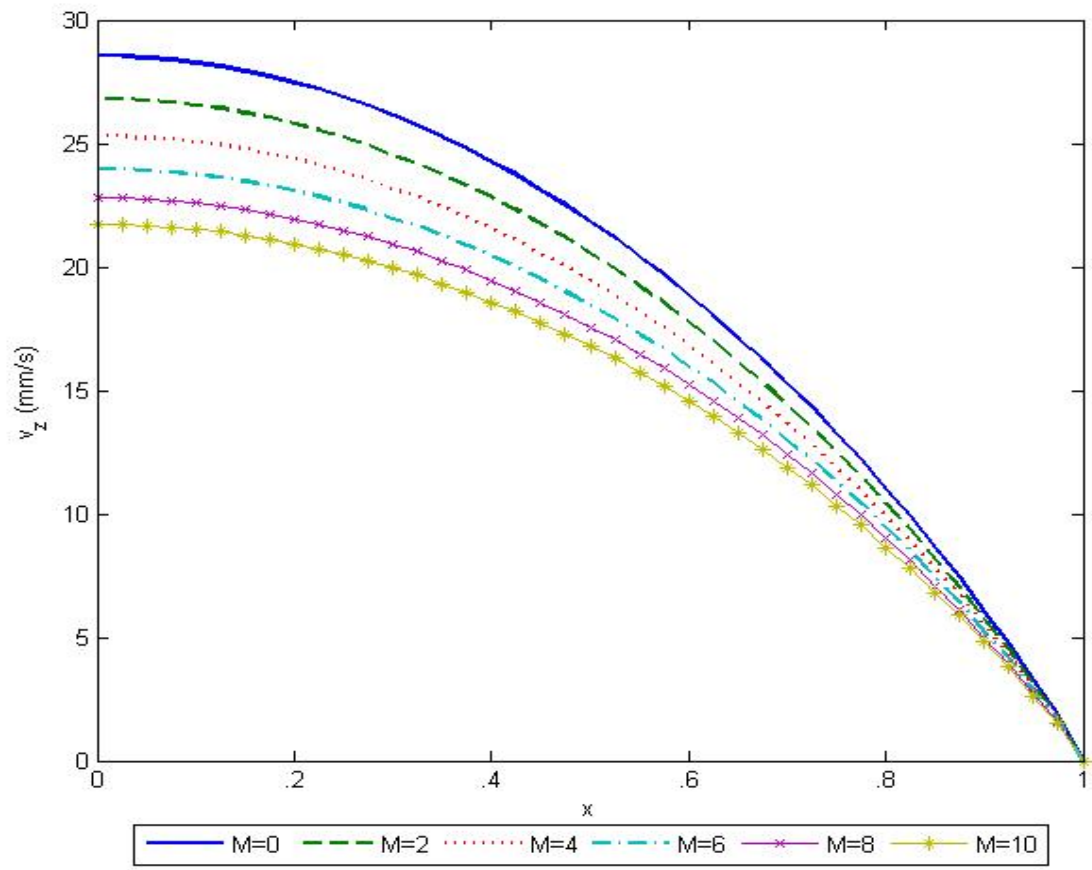

Figure 8. Axial velocity profiles for different magnetic field at $\mathrm{z}=23 \mathrm{~mm}$ $\left(\mathrm{T}_{\mathrm{m}}=0.4 \mathrm{a}, \mathrm{d}=13 \mathrm{~mm}, \mathrm{l}_{0}=16 \mathrm{~mm}\right)$ 
The variation of the time dependent wall shear stress at a specific location of $\mathrm{z}=23 \mathrm{~mm}$ for various magnetic field intensity has been shown in Figure 13. The stress yields all time higher values for an artery without applied magnetic field than the artery having applied magnetic field. The values are negative by direction

Figure 14 illustrates the variation of wall shear stress .for several values of the frequency and the amplitude of pressure gradient. The wall shear stress becomes more negative for small value of $\mathrm{f}_{\mathrm{p}}$ and an early separation can be noticed for large value of $\mathrm{f}_{\mathrm{p}}$. The wall shear stress is pulsatile in nature for all cases. The flow separation decreases by increasing the value of $A_{0}$.

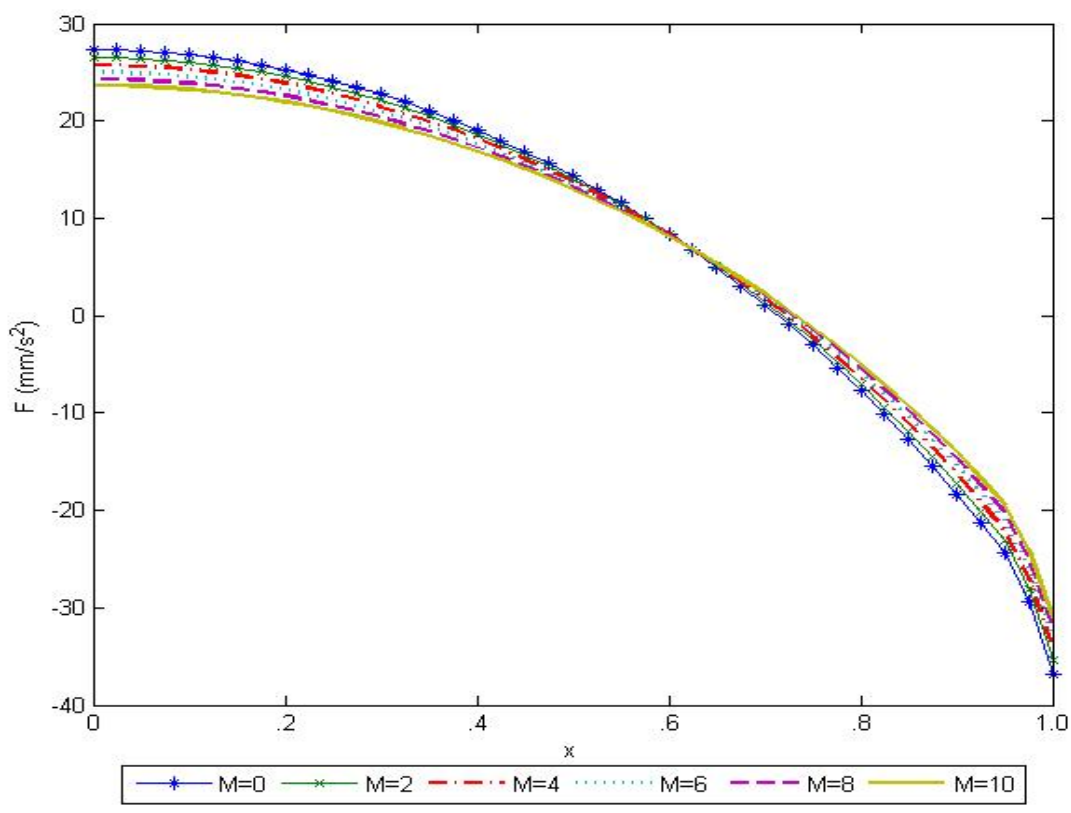

Figure 9. Radial variation of fluid acceleration at $\mathrm{t}=0.6 \mathrm{sec}$. $\left(\mathrm{a}_{0}=100 \mathrm{~mm} / \mathrm{s}^{2}, \mathrm{z}=23 \mathrm{~mm}, \mathrm{~T}_{\mathrm{m}}=0.4 \mathrm{a}, \mathrm{d}=13 \mathrm{~mm}, \mathrm{l}_{0}=16 \mathrm{~mm}\right)$

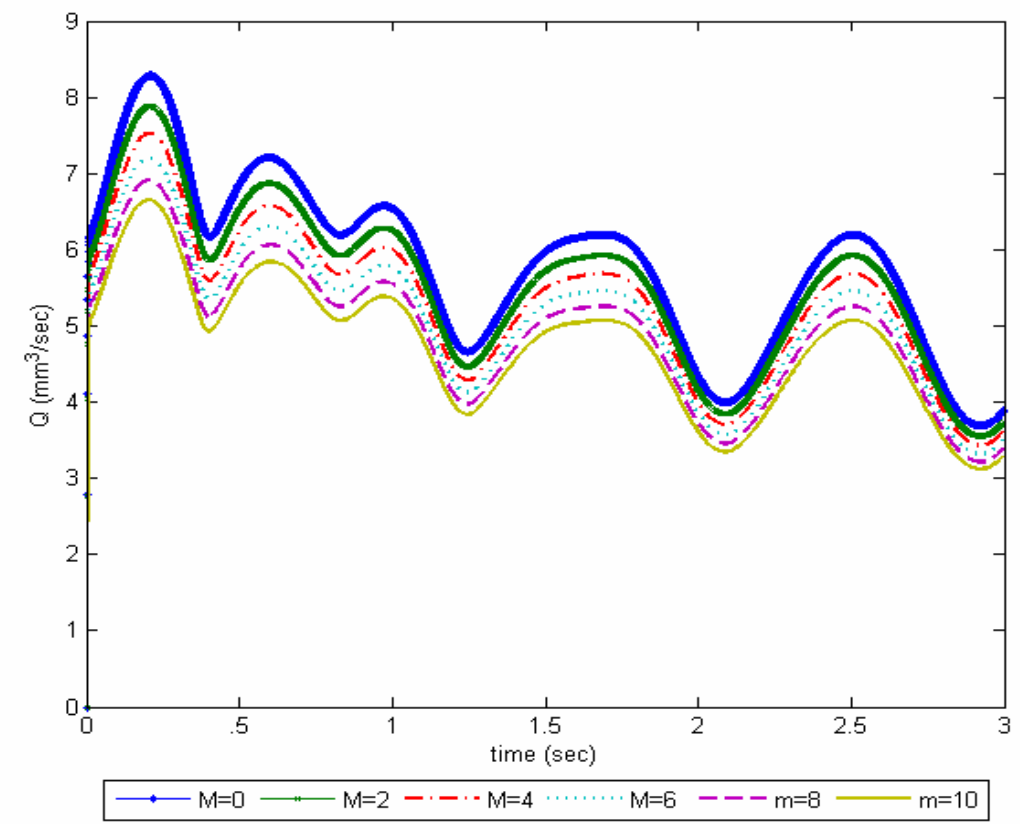

Figure 10. variation of the rate of flow with time at $\mathrm{z}=23 \mathrm{~mm}$ $\left(\mathrm{T}_{\mathrm{m}}=0.4 \mathrm{a}, \mathrm{d}=13 \mathrm{~mm}, \mathrm{l}_{0}=16 \mathrm{~mm}\right)$ 


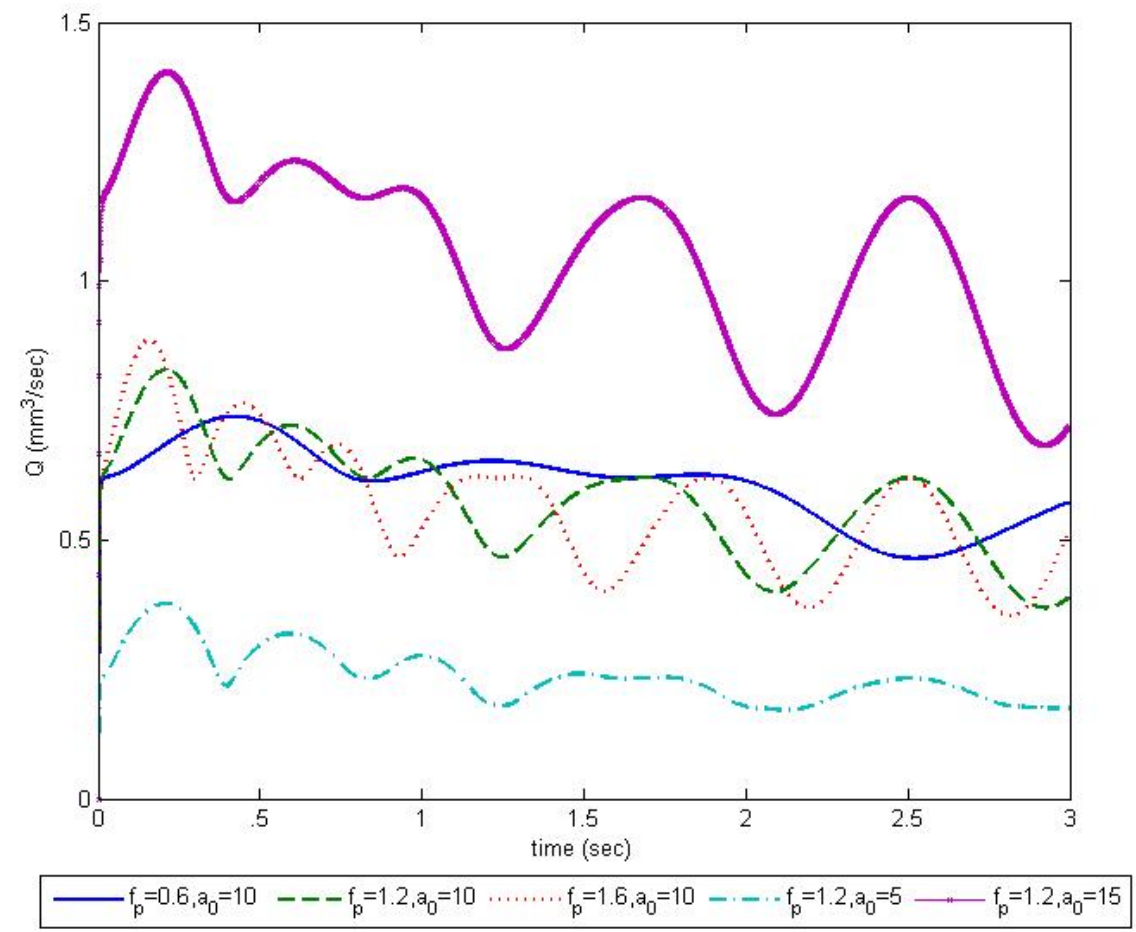

Figure 11. Variation of the rate of flow with time for different $\mathrm{f}_{\mathrm{b}}$ and $\mathrm{a}_{0}$ at $\mathrm{z}=23 \mathrm{~mm}$ $\left(\mathrm{f}_{\mathrm{p}}=1.2 \mathrm{~Hz}, \mathrm{a}_{0}=100 \mathrm{~mm} / \mathrm{s}^{2}, \mathrm{M}=2\right)$

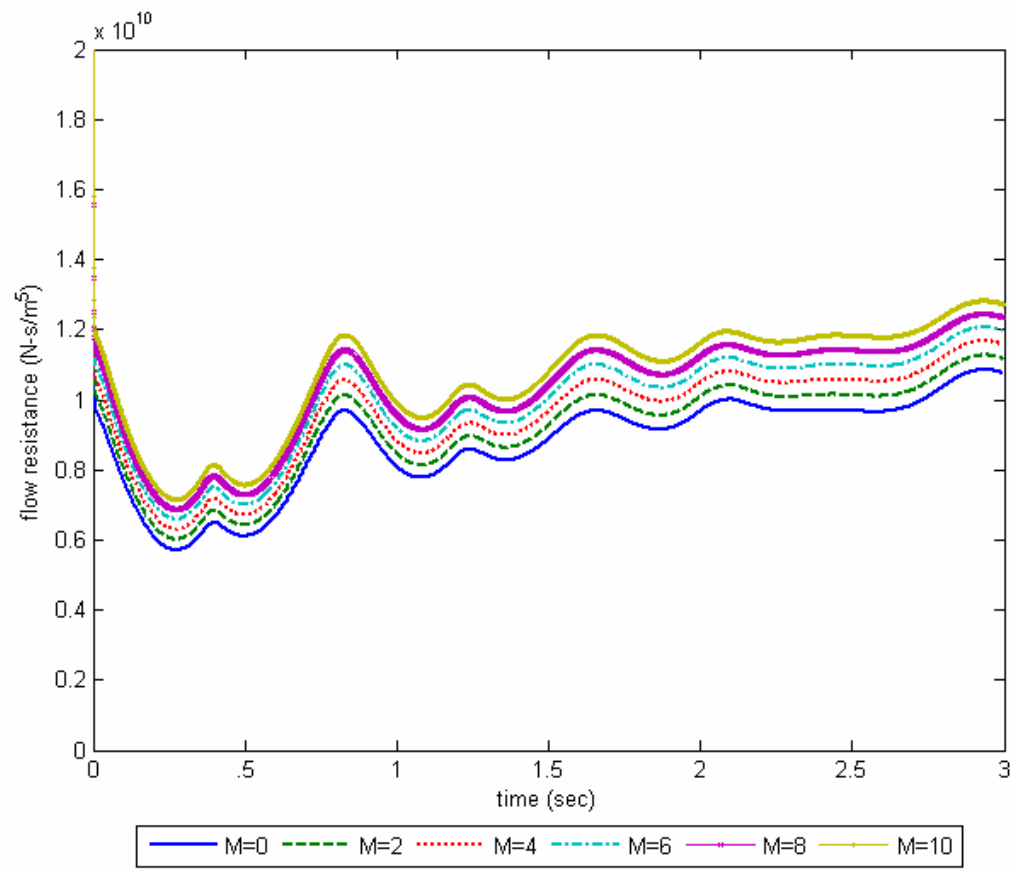

Figure 12. Variation of the resistance of flow with time for different magnetic field at $\mathrm{z}=23 \mathrm{~mm}$ $\left(\mathrm{f}_{\mathrm{p}}=1.2 \mathrm{~Hz}, \mathrm{a}_{0}=100 \mathrm{~mm} / \mathrm{s}^{2}\right)$ 


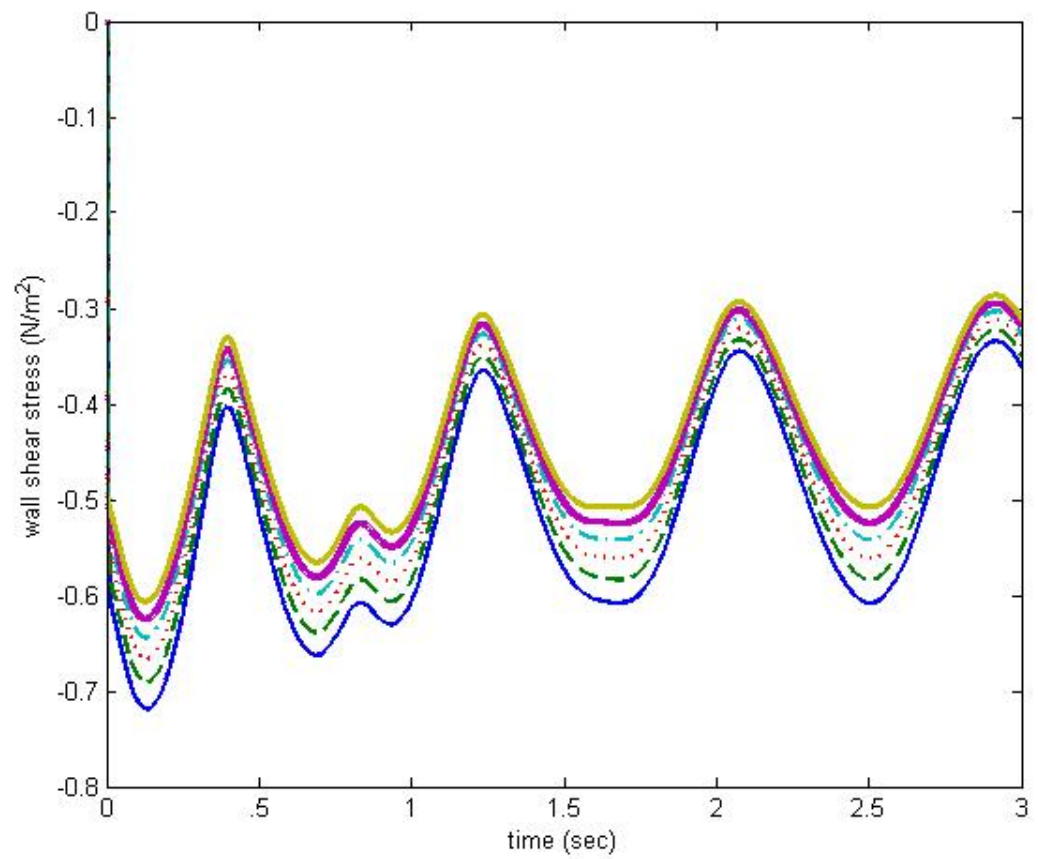

Figure 13. Variation of the wall shear stress with time at $\mathrm{z}=23 \mathrm{~mm}$ $\left(\mathrm{T}_{\mathrm{m}}=0.4 \mathrm{a}, \mathrm{d}=13 \mathrm{~mm}, \mathrm{l}_{0}=16 \mathrm{~mm}\right)$

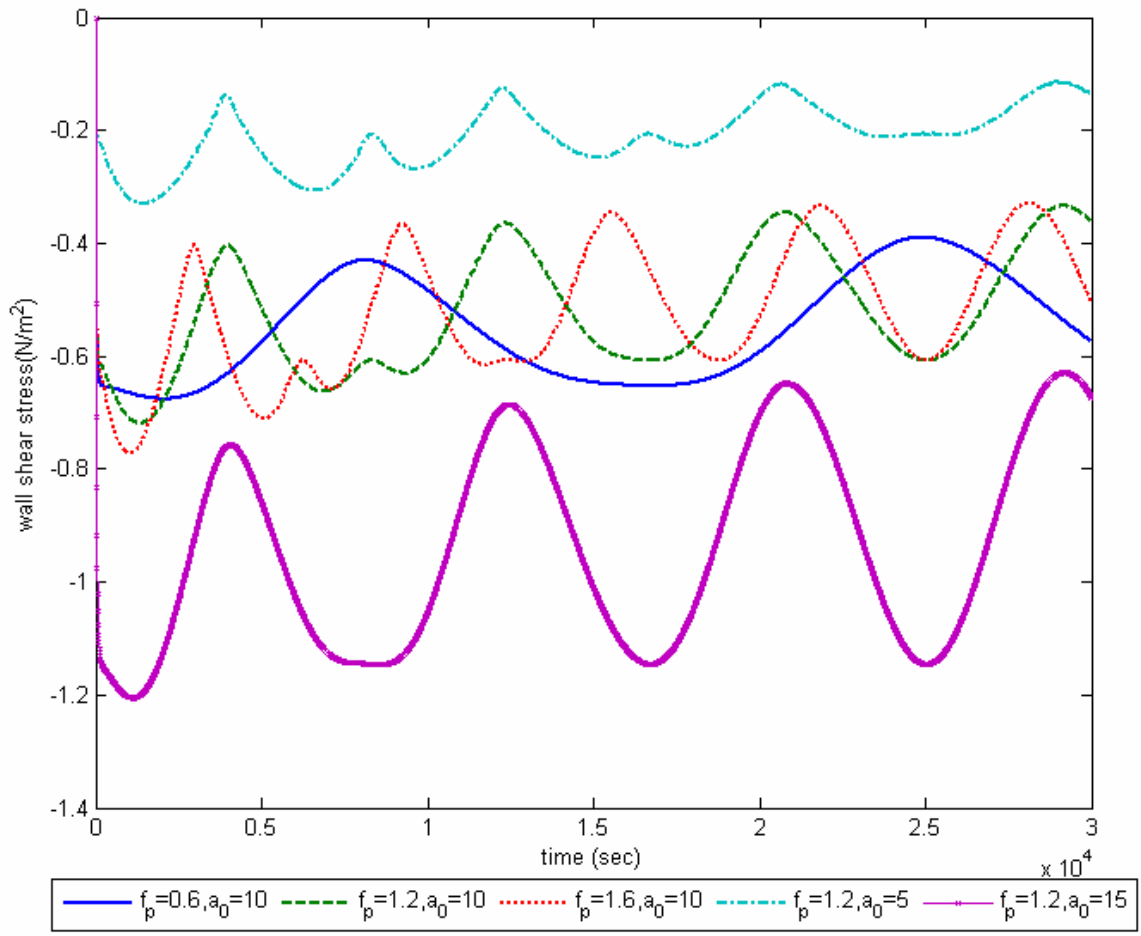

Figure 14. Variation of the wall shear stress with time for different $f_{\mathrm{p}}$ and $\mathrm{a}_{0}$ at $\mathrm{z}=28 \mathrm{~mm}$ $\left(\mathrm{T}_{\mathrm{m}}=0.4 \mathrm{a}, \mathrm{d}=13 \mathrm{~mm}, \mathrm{l}_{0}=16 \mathrm{~mm}, \mathrm{M}=2\right)$ 


\section{Conclusion}

This analysis presented numerical results for an unsteady blood flow in an artery with multiple stenosis, using the generalized power law model of blood viscosity under the influence of applied magnetic field. In this paper we used the time dependent radius of the artery having multiple stenosis, which is an important factor considered in this paper. The advantage of this study is that here we calculated effect of magnetic field on various fluid parameters like blood velocity, flow rate, wall shear stress, flow resistance and flow acceleration with the presence of multiple stenosis in the artery. All the flow characteristics are found to be affected by the influence of applied magnetic field as well as presence of multiple stenosis. Various physiological problems due to the back flow and low shear stress can be caused by the sufficiently large applied magnetic field. The present study would be helpful to analyze the effects of magnetic field on the human by various medical therapies like MRI, Catheter insertion and use of many devices e.g. cellular phone.

\section{Acknowledgement}

One of the authors Gaurav Varshney is thankful to Dr. M. S. Rautela, Principal, Government Degree College Karanprayag, Chamoli, India for his guidance, support and providing all facilities in the College for the preparation of this Manuscript.

\section{References}

Bali, R. and Awasthi, U. 2007. Effect of magnetic field on the resistance to blood flow through stenotic artery. Applied Mathematics and Computation, Vol. 188, pp. 1635-1641.

Buchanan Jr., J. R., Kleinstreuer, C. and Corner J. K. 2000. Rheological effects on pulsatile hemodynamics in a stenosed tube. Computers \& Fluids, Vol. 29, pp. 695-724.

Chakravarty, S. 1987. Effects of stenosis on the flow behaviour of blood in an artery. International Journal of Engineering Science, Vol. 25, No. 8, pp. 1003-1016.

Chakravarty, S. and Datta, A. 1992. Pulsatile blood flow in a porous stenotic artery. Mathl. Comput. Modelling, Vol. 16, No. 2, pp. 35-54.

Chakravarty, S. and Mandal, P. K. 1994. Mathematical modeling of blood flow through an overlapping arterial stenosis. Mathl. Comput. Modelling, Vol. 19, No. 1, pp. 59-70.

Chester, W. 1957. The effect of a magnetic field on Stokes flow in a conducting fluid. J. Fluid Mech,.Vol. 3, pp. $304-308$.

Deplano, V. and Siouffi, M. 1999. Experimental and Numerical study of pulsatile flow through a tapered artery with stenosis. Journal of Biomechanics, Vol. 32, pp. 1081-1090.

Gerrard, J. H. and Taylor, L. A. 1977. Mathematical model representing blood flow in arteries. Med. \& Biol. Eng. and Comput., Vol. 15, pp. 611-617.

Gmitrov, J. 2007. Static magnetic field effect on the arterial baroreflex-mediated control of microcirculation: implications for cardiovascular effects due to environmental magnetic fields. Radiat. Environ. Biophys., Vol. 46, pp. 281-290.

Haldar, K. 1985. Effects of the shape of stenosis on the resistance to blood flow through an artery. Bulletin of Mathematical Biology, Vol. 47, No. 4, pp. 545-550.

Hernan, A. and Gonzalez, R. 2007. Numerical implementation of viscoelastic blood flow in a simplified arterial geometry. Medical Engineering and Physics, Vol. 29, pp. 491-496.

Ismail, Z., Abdullah, I., Mustapha, N. and Amin, N. 2007. A power-law model of blood flow through a tapered overlapping stenosed artery. Applied Mathematics and Computation, Vol. 195, No. 2, pp. 669-680.

Jauchem, J.R. 1997. Exposure to extremely-low-frequency electromagnetic fields and radiofrequency radiation: cardiovascular effects in humans, Review Int Arch Occup Environ Health, Vol. 70, pp. 9-21.

Kaazempur - Mofrad, M., Wada, S., Myers, J. G. and Ethier, C. R. 2005. Mass transport and fluid flow in stenotic arteries: axisymmetric and asymmetric models. International Journal of Heat and Mass Transfer, Vol. 48, pp. 4510-4517.

Katiyar, V. K. and Basavarajappa, K.S. 2002. Blood flow in the cardiovsular system in the presence of magnetic field. International Journal of Applied Science and Computations, Vol. 9, No. 3, pp. 118-127.

Khanafer, K. M., Gadhoke, P., Berguer, R. and Bull, J. L. 2006. Modeling pulsatile flow in aortic aneurysms. Biorheology, Vol. 43, pp. 661-679.

Kinouchi, Y., Yamaguchi, H., and Tenforde, T.S. 1996. Theoretical analysis of magnetic field interactions with aortic blood flow. Bioelectromagnetics, Vol. 17, pp. 21-32.

Kuipers, N. T., Sauder, C.L. and Ray, C. A. 2007. Influence of static magnetic fields on pain perception and sympathetic nerve activity in humans. J. Appl. Physiol., Vol. 102, pp. 1410-1415.

Kumar, A., Varshney, C.L. and Sharma, G.C. Performance modeling and analysis of blood flow in elastic arteries. Applied Mathematics and Mechanics, Vol. 26, No. 3, pp. 345-354.

Liepsch, D. 2002. An introduction to biofluid mechanics - basic models and applications. Journal of Biomechanics, Vol. 35, pp. 415-435.

Liu, G. T., Wang, X. J., Ai, B. Q. and Liu, L. G. 2004. Numerical study of pulsatile flow through a tapered artery with stenosis. Chinese Journal of Physics, Vol. 42, No. 4-I, pp. 401-409.

Mandal, P. K. 2005. An unsteady analysis of non-Newtonian blood flow through tapered arteries with a stenosis. International Journal of NonLinear Mechanics, Vol. 40, pp. 151-164.

McKay, J.C., Prato, F.S. and Thomas, A.W. 2007. A literature review: the effects of magnetic field exposure on blood flow and blood vessels in the microvasculature. Bioelectromagnetics, Vol. 28, pp. 81-98. 
Prakash, J., Makinde, O. D. and Ogulu, A. 2004. Magnetic effect on oscillary blood flow in a constricted tube. Botswana Journal of Technology April , pp. 45-50.

Shalman. E., Rosenfeld, M., Dgany, E. and Einav, S. 2002. Numerical modeling of the flow in stenosed coronary artery: the relationship between main hemodynamic parameters. Computers in Biology and Medicine, Vol. 32, pp. 329-344.

Sud, V. K. and Sekhon, G. S. 1989. Blood flow through the human arterial system in the presence of a steady magnetic field. Phys. Med. Biol. Vol. 34, No. 7, pp. 795-805.

Tashtoush, B. and Magableh, A. 2008. Magnetic field effect on heat transfer and fluid flow charecteristics of blood flow in multi-stenotic arteries. Heat and Mass Transfer, Vol. 44, No. 3, pp. 297-304.

Tzirtzilakis, E. E. 2005. A mathematical model for blood flow in magnetic field. Physics of Fluids, Vol. 17:077103, pp. 1-15.

Villoresi, G., Kopytenko, Y. A.., Ptitsyna, N. G., Tyasto, M. I., Kopytenko, E. A, Iucci, N. and Voronov, P. M. 1994. The influence of geomagnetic storms and man made magnetic field disturbances on the incidence of myocardial infarction in St. Petersburg (Russia). Phys. Med., Vol. 10, pp. 107-117.

\section{Biographical notes}

Gaurav Varshney received Ph.D. from Indian Institute of Technology Roorkee, India in 2009. He is Assistant Professor in the Department of Mathematics, Government Degree College Karanprayag, Chamoli, India. He has published 5 research papers in referred international journals and presented more than 15 research article in National and International Conferences. His area of research includes Bio-fluid Dynamics, Mathematical Modeling, Respiratory Mechanics, Numerical Solutions of ODE and PDE.

Dr. V. K. Katiyar is a Professor in the Department of Mathematics, Indian Institute of Technology Roorkee, India. He has more than 30 years of experience in teaching and research. His current area of research includes Biomechanics, Mathematical Modeling, Respiratory Mechanics, Heat and Mass Transfer, Industrial Mathematics. He has published more than One hundred papers in referred international journals. He has also presented more than one hundred research articles in national and international conferences and organized many National and International Conferences.

Sushil Kumar received M.Sc. and Ph.D. from Indian Institute of Technology Roorkee, India in 2001 and 2007 respectively. He is Assistant Professor in the Department of Mathematics, S. V. National Institute of Technology Surat, India. He has published 5 research papers in referred international journals. His area of research includes Heat and Mass Transfer, Computational Fluid Dynamics, Mathematical Modeling, Numerical Solutions of ODE and PDE.

Received March 2010

Accepted April 2010

Final acceptance in revised form April 2010 